\title{
Different Characteristics of the Corticospinal Tract According to the Cerebral Origin: DTI Study
}

J.P. Seo and S.H. Jang

\begin{abstract}
BACKGROUND AND PURPOSE: Little is known about differences in corticospinal tract fibers according to cerebral origin. Using diffusion tensor tractography, we attempted to investigate the characteristics of the CST according to the cerebral origin in the human brain.
\end{abstract}

MATERIALS AND METHODS: Thirty-six healthy subjects were recruited for this study. A 1.5T Gyroscan Intera system was used for acquisition of DTI. CSTs were reconstructed by selection of fibers passing through seed and target ROls: seed ROls, the area of the CST at the pontomedullary junction; target ROIs, the primary motor cortex, the primary somatosensory cortex, the dorsal premotor cortex, and the supplementary motor area.

RESULTS: A significant difference in tract volume was observed in each ROI ( $P<.05)$ : M1 (2373.6, 36.9\%), S1 (2037.7, 31.7\%), SMA (1588.0, $24.7 \%)$, and dPMC $(429.8,6.7 \%)$. Regarding fractional anisotropy values, the dPMC or SMA showed higher values than the M1 or S1; however, the opposite occurred in terms of the mean diffusivity value $(P<.05)$. In addition, fractional anisotropy and mean diffusivity values of the dPMC differed from those of the SMA $(P<.05)$; in contrast, no significant difference was observed between the M1 and S1 $(P>.05)$.

CONCLUSIONS: Tract volume was found to differ according to cerebral origin and was, in descending order, M1, S1, SMA, and dPMC. In addition, the directionality and diffusivity of CST fibers in the SMA and the APMC differed from those of the M1 and S1, which showed similar characteristics.

ABBREVIATIONS: $\mathrm{CST}=$ corticospinal tract; $\mathrm{dPMC}=$ dorsal premotor cortex; $\mathrm{Ml}=$ primary motor cortex, $\mathrm{Sl}=$ primary somatosensory cortex; $\mathrm{SMA}=$ supplementary motor area

$\mathbf{T}$ he corticospinal tract is a major neural tract for motor function in the human brain. ${ }^{1-5}$ The CST is known be involved mainly in movement execution of distal extremities, particularly fine-motor activities of the hand. ${ }^{1-5}$ The CST originates from various cortical areas, such as the secondary motor area, the parietal cortex, and the primary motor cortex. The multiple cerebral origins of the CST appear to be important in terms of multiple functions of CST fibers and motor recovery mechanisms: perilesional reorganization following M1 injury. ${ }^{6-10}$ Many previous studies have reported differences in characteristics of CST fibers, in terms

Received July 2, 2012; accepted after revision September 17.

From the Department of Physical Medicine and Rehabilitation, College of Medicine, Yeungnam University, Taegu, Republic of Korea.

This work was supported by the Basic Science Research Program through the National Research Foundation of Korea funded by the Ministry of Education, Science and Technology (2012R1A1A4A01001873).

Please address correspondence to Sung Ho Jang, MD, Department of Physical Medicine and Rehabilitation, College of Medicine, Yeungnam University 317-1, Daemyungdong, Namku, Taegu, 705-717, Republic of Korea; e-mail: strokerehab@hanmail.net

-- Indicates open access to non-subscribers at www.ajnr.org

http://dx.doi.org/10.3174/ajnr.A3389 of the amount of CST fibers and function, according to the origin of the cerebral cortex. ${ }^{11-18}$ Most of these studies have used animal brains because only postmortem histologic studies or microelectrode stimulation studies have been used for research on the human brain. ${ }^{11,14-17}$

By contrast, diffusion tensor tractography, a technique derived from DTI, allows $3 \mathrm{D}$ visualization and estimation of the CST. ${ }^{19-21}$ Several diffusion tensor tractography studies have reported the characteristics of the whole $\mathrm{CST}^{22-24}$; however, little is known about differences in CST fibers according to the cerebral origin. $^{17}$

In the current study, by using diffusion tensor tractography, we attempted to investigate the characteristics of the CST according to the cerebral origin in the human brain.

\section{MATERIALS AND METHODS \\ Subjects}

We recruited 36 healthy right-handed subjects (mean age, 34.2 years; range, $20 \sim 49$ years; male, 18 subjects [mean age, 33.5 years; range, $20 \sim 48$ years]; female, 18 subjects [mean age, 34.9 years; range, $20 \sim 49$ years]) with no previous history of neurologic, psy- 
chiatric, or physical illness. All subjects understood the purpose of the study and provided written informed consent before participation. The study protocol was approved by our local institutional research board.

\section{Diffusion Tensor Image}

DTI data were obtained by using a Synergy-L sensitivity encoding head coil on a $1.5 \mathrm{~T}$ Gyroscan Intera system (Philips, Best, the Netherlands) equipped with single-shot echo-planar imaging. For each of the 32 noncollinear diffusion-sensitizing gradients, we acquired 67 contiguous sections parallel to the anterior/posterior commissure line. Imaging parameters were as follows: acquisition matrix $=96 \times 96$, reconstructed to matrix $=128 \times 128$, FOV $=$ $221 \times 221 \mathrm{~mm}^{2}, \mathrm{TR}=10,726 \mathrm{~ms}, \mathrm{TE}=76 \mathrm{~ms}$, parallel imaging reduction factor (sensitivity encoding factor) $=2$, EPI factor $=$ $49, b=1000 \mathrm{~s} / \mathrm{mm}^{2}, \mathrm{NEX}=1$, and a section thickness of $2.3 \mathrm{~mm}$ (acquired isotropic voxel size, $2.3 \times 2.3 \times 2.3 \mathrm{~mm}^{3}$ ).

\section{Fiber Tracking}

The Oxford Centre for Functional Magnetic Resonance Imaging of the Brain (FMRIB) Software Library (www.fmrib.ox.ac.uk/fsl) was used for analysis of diffusion-weighted imaging data. Affine multiscale $2 \mathrm{D}$ registration was used for correction of head motion effect and image distortion due to eddy currents. A probabilistic tractography method, based on a multifiber model, was used in performance of fiber tracking, which was applied in the present study using tractography routines implemented in FMRIB Diffusion (5000 streamline samples, 0.5 -mm step lengths, curvature thresholds $=0.2) .^{25-27}$

CSTs for the M1, the primary somatosensory cortex, dorsal premotor cortex, and supplementary motor area were determined by selection of fibers passing through seed and target ROIs. Seed ROIs were placed at the CST portion of the pontomedullary junction on the color map. Placement of target ROIs was as follows: 1) the primary motor cortex (anterior boundary: precentral sulcus; posterior boundary: central sulcus; medial boundary: the midline between the right and left hemispheres; lateral boundary: the line passing through the lateral margin of the precentral knob and horizontal to the midline); 2) the primary somatosensory cortex (anterior boundary: central sulcus; posterior boundary: postcentral sulcus; medial boundary: the midline between the right and left hemispheres; lateral boundary: the line passing through the lateral margin of the precentral knob and horizontal to the midline between the right and left hemispheres); 3 ) the dorsal premotor cortex (anterior boundary: the line joining the anterior extent of the SMA; posterior boundary: precentral sulcus; medial boundary: the lateral margin of the SMA; lateral boundary: the line passing through the lateral margin of the precentral knob and horizontal to the midline); 4) the supplementary motor area (anterior boundary: the line drawn through the anterior commissure perpendicular to the anterior/posterior commissure line; posterior boundary: anterior margin of M1; medial boundary: midline between the right and left hemispheres; lateral boundary: the line $10.6 \%$ lateral from the midline, the ratio of $15 \mathrm{~mm}$ to the maximum width of the Montreal Neurological Institute atlas) (Fig 1). 9,28,29 Of 5000 samples generated from each seed voxel, results for each contact were visualized thresholds and weightings of tract probability at a minimum of 1 streamline through each voxel for analysis. Values of fractional anisotropy, mean diffusivity, and tract volume of the CSTs were measured.

\section{Statistical Analysis}

The Statistical Package for the Social Sciences software (Version 15.0; SPSS, Chicago, Illinois) was used for data analysis. Using 1-way ANOVA with the least significant difference post hoc test, we determined the differences in values for tract volume, fractional anisotropy, and mean diffusivity among CSTs from the M1,

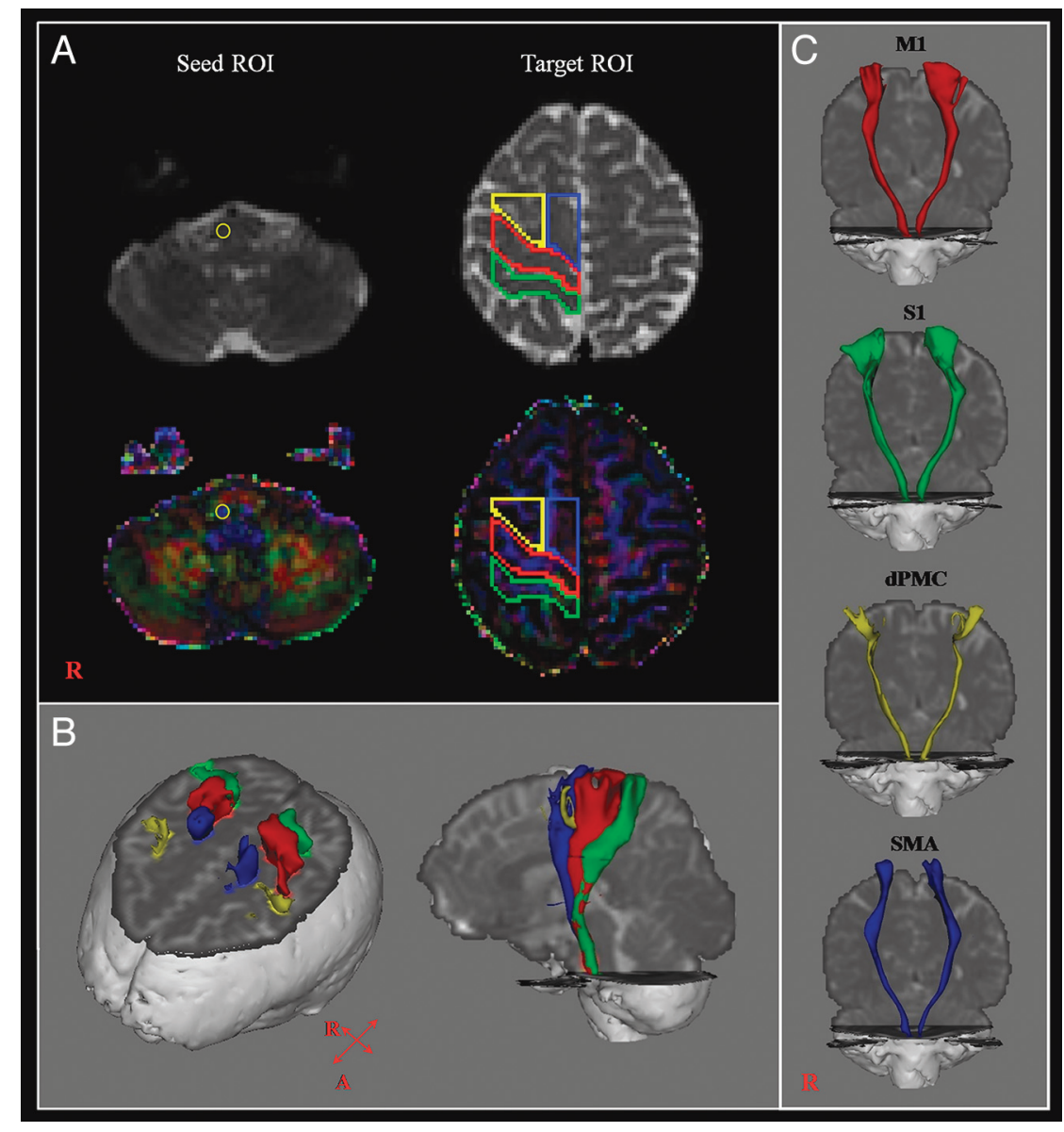

FIG 1. A, The seed ROI was placed on the pontomedullary junction (CST area). Target ROls were placed on the primary motor cortex (red), the primary somatosensory cortex (green), the dorsal premotor cortex (yellow), and the supplementary motor area (blue). B, CSTs were reconstructed in both hemispheres. Red indicates CSTs for the primary motor cortex; green, CSTs for the primary somatosensory cortex; yellow, CSTs for the dorsal premotor cortex; blue, CSTs for the supplementary motor area. C, CSTs from each ROI were reconstructed in both hemispheres. 


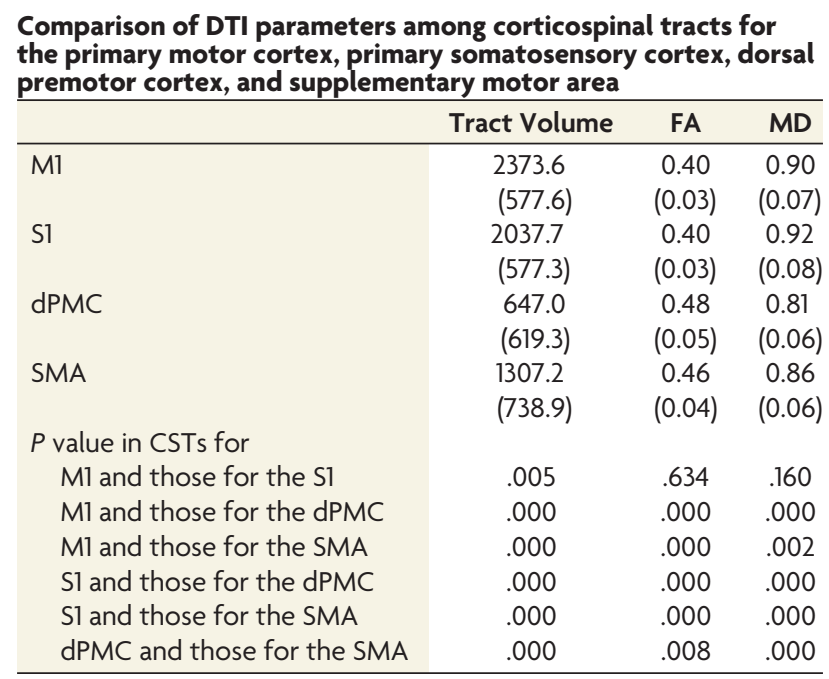

Note:-FA indicates fractional anisotropy; MD, mean diffusivity, $M D \times 10^{-3}\left(\mathrm{~mm}^{2} / \mathrm{s}\right)$. Values represent mean ( \pm SD); post hoc least significant difference test was used for comparisons of DTI parameters.

S1, dPMC, and SMA. The independent $t$ test was used for determination of the difference in values of tract volume, fractional anisotropy, and mean diffusivity from each CST between the right and left hemispheres. The significance level for the $P$ value was set at .05 .

\section{RESULTS}

CSTs from the M1, S1, and SMA were reconstructed in 72 hemispheres of 36 healthy subjects. By contrast, CSTs from the dPMC were reconstructed in 56 (77.8\%) of 72 hemispheres. Therefore, we performed an analysis of data from CSTs having a cerebral origin for the 56 hemispheres; all CSTs were reconstructed.

A summary of mean values for tract volume, fractional anisotropy, and mean diffusivity of the CSTs is shown in the Table. Tract volumes differed significantly among each ROI $(P<.05)$ and were, in order, the following: M1 (2373.6, 37.3\%), S1 (2037.7, $32.0 \%)$, SMA $(1307.2,20.5 \%)$, and dPMC (647.0, 10.2\%). With regard to fractional anisotropy values, CSTs from the APMC were significantly higher than CSTs for the M1, S1, and SMA $(P<.05)$. In addition, CSTs from the SMA were significantly higher than CSTs for the M1 and S1 $(P<.05)$. However, no significant differences were observed between CSTs for the M1 and S1 $(P>.05)$. As for mean diffusivity values, CSTs from the dPMC were significantly lower than CSTs for the M1, S1, and SMA $(P<.05)$. CSTs from the SMA were also significantly lower than CSTs for the M1 and S1 $(P<.05)$. By contrast, no significant difference was observed between CSTs from the M1 and S1 $(P>.05)$.

In comparison with the DTI parameters between hemispheres, fractional anisotropy values of the CSTs for the M1 and $\mathrm{S} 1$ of left hemisphere were significantly higher than those of the right hemisphere $(P<.05)$. By contrast, mean diffusivity values of the CSTs for the M1, S1, and dPMC of the right hemisphere were significantly higher than those of left hemisphere $(P<.05)$. Tract volume of the CSTs from the SMA of the left hemisphere was significantly higher than those of the right hemisphere $(P<.05)$.

\section{DISCUSSION}

In the current study, we investigated the characteristics of the CST according to the cerebral origin. We found the following: 1) Tract volumes differed significantly in each ROI, in the following order: M1 (37.3\%), S1 (32.0\%), SMA (20.5\%), and APMC (10.2\%). 2) Regarding fractional anisotropy values, the dPMC or SMA showed higher values than the M1 or S1; however, the opposite was true in terms of mean diffusivity values. In addition, fractional anisotropy and mean diffusivity values of the dPMC differed significantly from those of the SMA. In contrast, no significant difference in either value was observed between the M1 and S1. Tract volume is determined by counting the number of voxels contained within a neural tract. ${ }^{30}$ Consequently, an increment of tract volume may indicate the increment of fiber number in a neural tract. Our results for tract volume suggest that the number of CST fibers is the same as the order of the tract volume.

On the other hand, fractional anisotropy value indicates the degree of directionality of water diffusion and has a range of zero (completely isotropic diffusion) to 1 (completely anisotropic diffusion). ${ }^{31,32}$ It represents the white matter organization: In detail, the degree of directionality and integrity of white matter microstructures, such as axon, myelin, and microtubule, and mean diffusivity values, indicate the magnitude of water diffusion. ${ }^{31,32}$ Therefore, our results for values of fractional anisotropy and mean diffusivity appear to indicate that CST fibers from the dPMC or SMA have the characteristics of higher anisotropy and lower diffusivity than those of the M1 and S1; and CST fibers from the M1 and 1 have similar anisotropy and diffusivity. Characteristics of the S1, the second largest source of CST fibers, which has DTI characteristics that are similar to those of the M1, appear to have an association with the high incidence of reorganization into the S1 following M1 infarct in patients with stroke. ${ }^{10,33-35}$

Several previous studies have reported the distribution of the CST origin in the cerebral cortex in primates. ${ }^{11,12,14,17}$ In 1961, Russell and Demyer ${ }^{11}$ reported that in the macaque rhesus, 31\% of the descending fibers in the pyramid originated from the M1, $29 \%$ from the premotor cortex, and $40 \%$ from the parietal lobe. In 1994, in a retrograde transported fluorescent tracer-labeling study of the macaque, Galea and Darian-Smith ${ }^{14}$ found that the contralateral CST was projected mostly from area 4 (35\%), and also originated from the adjacent areas as follows; dPMC (6\%), SMA (15\%), postarcuate cortex $(2.6 \%)$, cingulate cortex $(10 \%)$, and parietal cortex (3a: 2.2\%; 3b/1: 9\%; 2/5: 13\%; secondary somatosensory cortex and insula: $3.4 \%$ ). With regard to the human brain, Jane et al in $1967^{12}$ reported that $60 \%$ of CST fibers originated from the precentral gyrus and that the remaining fibers originated from the premotor cortex and parietal lobe. They estimated the amount of the CST in a patient who had shown involuntary movements in the left upper and lower extremities since 2.5 years of age. She underwent ablation of the right precentral gyrus 2 times at 27 and 31 years of age. When she died at 51 years of age due to a left intracerebral hemorrhage, they found that the amount in the right pyramid was $40 \%$ of the left pyramid. As a result, they reported that $60 \%$ of the CST originated from the precentral gyrus.

Kumar et al in $2009^{17}$ recently reported on the origin of the CST by using diffusion tensor tractography. DTI was performed 
in 42 healthy children (2.6-12.7 years of age). They reconstructed the CST by placement of 2 ROIs (pons and corona radiata) and found that the CST originated from both the pre- and postcentral gyri (71.4\%), the precentral gyrus only (19\%), and the postcentral gyrus only $(7.1 \%)$. By contrast, because reconstruction by using diffusion tensor tractography in the cerebral cortex is not accurate, we placed ROIs in the cerebral cortex instead of the corona radiata.

Many previous studies have reported the different functions and characteristics of the CST according to the cerebral origin. ${ }^{6-9,15,16}$ However, a few studies have reported the anatomic difference between the M1 and SMA. ${ }^{16,36,37}$ In 1996, Dum and Strick $^{36}$ investigated the pattern of spinal termination of efferents from the SMA and M1 by using an anterograde transport method in macaque monkeys. They found that the extent and attenuation of the M1 labeling were greater than those in the SMA. Subsequently, Maier et al in $1997^{37}$ demonstrated that the projections from the M1 occupied $21 \%-65 \%$ and those from the SMA, $1 \%-6 \%$ by using densitometric analysis in the macaque monkey. In 2002, the same research group investigated differences in hand representations of the M1 and SMA by using the same method in the macaque monkey. ${ }^{16}$ They reported that corticospinal projections from the M1 showed far greater attenuation and occupied a much greater proportion of hand muscle motor nuclei than those of the SMA.

However, many studies have reported differences in function of the CST according to the cerebral origin, as follows ${ }^{6-9}: \mathrm{M} 1$, execution of movement; SMA, planning and coordination of internally generated movement; PMC, involved in visually guided movements; and somatosensory cortex, descending control of sensory afferent input generated by movements. These different functions appear to have an association with different clinical manifestations, such as weakness, apraxia, and impaired sensory coordination.

\section{CONCLUSIONS}

We found that the tract volume differed according to cerebral origin: M1 (37.3\%), S1 (32.0\%), SMA (20.5\%), and dPMC $(10.2 \%)$. In addition, DTI characteristics of CST fibers differed according to the cerebral origin: The directionality and diffusivity of CST fibers from the SMA and APMC differed from those of the M1 and S1, which showed similar characteristics.

In this study, several limitations should be considered. We think that due to the different boundaries of ROIs, direct comparison of these results with those of previous studies is problematic. For ROIs of the M1, S1, and dPMC between the midline and lateral margin of the precentral knob, we excluded the lateral portion (lateral area of the $\mathrm{APMC}$, the ventral PMC, and the face somatotopy of the M1 and S1) from the lateral margin of the precentral knob because its reconstruction might not be accurate, for the following reasons: 1) The angle from the lateral area from the lateral margin of the precentral knob was too steep, and 2) the crossing-fiber effect of the superior longitudinal fasciculus and arcuate fasciculus can hinder exact reconstruction. By contrast, we included all SMA proper. This might cause underestimation of the relative numbers of CST fibers of the M1, S1, and APMC, compared with other cerebral areas.
In addition, in interpretation of our results, the limitations of DTI should be considered. Although diffusion tensor tractography is a powerful anatomic imaging tool, with the capacity to demonstrate gross fiber architecture, it has several limitations in reconstruction of neural tracts. ${ }^{38-41}$ In particular, regions of fiber complexity and crossing can prevent full reflection of the underlying fiber architecture by diffusion tensor tractography. Therefore, we think that further studies to overcome these limitations are necessary.

\section{REFERENCES}

1. Nathan PW, Smith MC. Long descending tracts in man. I. Review of present knowledge. Brain 1955;78:248-303

2. Nyberg-Hansen R, Rinvil E. Some comments on the pyramidal tract, with special reference to its individual variations in man. Acta Neurol Scand 1963;39:1-30

3. York DH. Review of descending motor pathways involved with transcranial stimulation. Neurosurgery 1987;20:70-73

4. Davidoff RA. The pyramidal tract. Neurology 1990;40:332-39

5. Jang SH. The role of the corticospinal tract in motor recovery in patients with a stroke: a review. NeuroRehabilitation 2009;24:285-90

6. Rizzolatti G, Luppino G, Matelli M. The organization of the cortical motor system: new concepts. Electroencephalogr Clin Neurophysiol 1998;106:283-96

7. Rizzolatti G, Luppino G. The cortical motor system. Neuron 2001; 31:889-901

8. Lemon RN, Griffiths J. Comparing the function of the corticospinal system in different species: organizational differences for motor specialization? Muscle Nerve 2005;32:261-79

9. Mayka MA, Corcos DM, Leurgans SE, et al. Three-dimensional locations and boundaries of motor and premotor cortices as defined by functional brain imaging: a meta-analysis. Neuroimage 2006;31: 1453-74

10. Jang SH. Perilesional reorganization of motor function in stroke patients. Neural Regeneration Res 2010;5:1668-72

11. Russell JR, Demyer W. The quantitative corticoid origin of pyramidal axons of Macaca rhesus: with some remarks on the slow rate of axolysis. Neurology 1961;11:96-108

12. Jane JA, Yashon D, DeMyer W, et al. The contribution of the precentral gyrus to the pyramidal tract of man. J Neurosurg 1967;26: $244-48$

13. Toyoshima K, Sakai H. Exact cortical extent of the origin of the corticospinal tract (CST) and the quantitative contribution to the CST in different cytoarchitectonic areas: a study with horseradish peroxidase in the monkey. J Hirnforsch 1982;23:257-69

14. Galea MP, Darian-Smith I. Multiple corticospinal neuron populations in the macaque monkey are specified by their unique cortical origins, spinal terminations, and connections. Cereb Cortex 1994;4: 166-94

15. Lemon RN, Maier MA, Armand J, et al. Functional differences in corticospinal projections from macaque primary motor cortex and supplementary motor area. Adv Exp Med Biol 2002;508:425-34

16. Maier MA, Armand J, Kirkwood PA, et al. Differences in the corticospinal projection from primary motor cortex and supplementary motor area to macaque upper limb motoneurons: an anatomical and electrophysiological study. Cereb Cortex 2002;12:281-96

17. Kumar A, Juhasz C, Asano E, et al. Diffusion tensor imaging study of the cortical origin and course of the corticospinal tract in healthy children. AJNR Am J Neuroradiol 2009;30:1963-70

18. AI Masri O. An essay on the human corticospinal tract: history, development, anatomy, and connections. Neuroanatomy 2011;10: $1-4$

19. Mori S, Crain BJ, Chacko VP, et al. Three-dimensional tracking of axonal projections in the brain by magnetic resonance imaging. Ann Neurol 1999;45:265-69

20. Yamada K, Mori S, Nakamura H, et al. Fiber-tracking method re- 
veals sensorimotor pathway involvement in stroke patients. Stroke 2003;34:E159-62

21. Puig J, Pedraza S, Blasco G, et al. Wallerian degeneration in the corticospinal tract evaluated by diffusion tensor imaging correlates with motor deficit $\mathbf{3 0}$ days after middle cerebral artery ischemic stroke. AJNR Am J Neuroradiol 2010;31:1324-30

22. Kunimatsu A, Aoki S, Masutani Y, et al. The optimal trackability threshold of fractional anisotropy for diffusion tensor tractography of the corticospinal tract. Magn Reson Med Sci 2004;3:11-17

23. Reich DS, Smith SA, Jones CK, et al. Quantitative characterization of the corticospinal tract at 3T. AJNR Am J Neuroradiol 2006;27:2168-78

24. Frey D, Strack V, Wiener E, et al. A new approach for corticospinal tract reconstruction based on navigated transcranial stimulation and standardized fractional anisotropy values. Neuroimage 2012;62: 1600-09

25. Behrens TE, Johansen-Berg H, Woolrich MW, et al. Non-invasive mapping of connections between human thalamus and cortex using diffusion imaging. Nat Neurosci 2003;6:750-57

26. Smith SM, Jenkinson M, Woolrich MW, et al. Advances in functional and structural MR image analysis and implementation as FSL. Neuroimage 2004;23(suppl 1):S208-19

27. Behrens TE, Berg HJ, Jbabdi S, et al. Probabilistic diffusion tractography with multiple fibre orientations: what can we gain? Neuroimage 2007;34:144-55

28. Hansen JT, Koeppen BM, Netter FH, et al. Atlas of Neuroanatomy and Neurophysiology: Selections from the Netter Collection of Medical Illustrations. Icon Custom Communication; 2002

29. Yoshor D, Mizrahi E. Clinical Brain Mapping. New York: McGrawHill; 2012

30. Pagani E, Agosta F, Rocca MA, et al. Voxel-based analysis derived from fractional anisotropy images of white matter volume changes with aging. Neuroimage 2008;41:657-67
31. Assaf Y, Pasternak O. Diffusion tensor imaging (DTI)-based white matter mapping in brain research: a review. J Mol Neurosci 2008;34: $51-61$

32. Neil JJ. Diffusion imaging concepts for clinicians. J Magn Reson Imaging 2008;27:1-7

33. Cramer SC, Moore CI, Finklestein SP, et al. A pilot study of somatotopic mapping after cortical infarct. Stroke 2000;31:668-71

34. Jang SH, Cho SH, Kim YH, et al. Cortical activation changes associated with motor recovery in patients with precentral knob infarct. Neuroreport 2004;15:395-99

35. Jang SH, Ahn SH, Yang DS, et al. Cortical reorganization of hand motor function to primary sensory cortex in hemiparetic patients with a primary motor cortex infarct. Arch Phys Med Rehabil 2005;86: 1706-08

36. Dum RP, Strick PL. Spinal cord terminations of the medial wall motor areas in macaque monkeys. J Neurosci 1996;16:6513-25

37. Maier MA, Davis JN, Armand J, et al. Anatomical and electrophysiological comparison of corticomotoneuronal projections from primary motor cortex and supplementary motor area in the macaque monkey. J Physiol 1997;505(p):84

38. Lee SK, Kim DI, Kim J, et al. Diffusion-tensor MR imaging and fiber tractography: a new method of describing aberrant fiber connections in developmental CNS anomalies. Radiographics 2005;25:5365, discussion 66-68

39. Parker GJ, Alexander DC. Probabilistic anatomical connectivity derived from the microscopic persistent angular structure of cerebral tissue. Philos Trans R Soc Lond B Biol Sci 2005;360:893-902

40. Yamada K. Diffusion tensor tractography should be used with caution. Proc Natl Acad Sci U S A 2009;106:E14, author reply E15

41. Yamada K, Sakai K, Akazawa K, et al. MR tractography: a review of its clinical applications. Magn Reson Med Sci 2009;8:165-74 